\title{
Conhecimento de gestantes de uma comunidade carente sobre os exames de detecção precoce do câncer cérvico-uterino e de
} mama*

\author{
Knowledge of pregnant women on the tests to early detect cervix uteri and \\ breast cancer in a poor community
}

Rosa Aurea Quintella Fernandes' e Nádia Zanon Narchi

\begin{abstract}
Resumo
0 câncer ginecológico apresenta um alto índice de morbi-mortalidade no Brasil, representando grave problema de saúde pública, apesar das campanhas informativas e da maior participação das mulheres na sociedade em busca da cidadania. A relevância do tema despertou 0 interesse na realização desse trabalho descritivo, exploratório e quantitativo, que teve por objetivo identificar o conhecimento de gestantes sobre os exames de detecção precoce do câncer cérvico-uterino e de mama. $0 \mathrm{~s}$ dados foram obtidos por meio de entrevista aplicada a 49 gestantes que participaram de grupos de promoção à saúde em uma comunidade carente do município de São Paulo. Os resultados revelaram que $75 \%$ das mulheres conhecem o exame colpocitológico e o associam à detecção de problemas no útero e que $63,3 \%$ colheu o exame há um ano ou menos havendo, em $29 \%$ dos casos, sido detectado algum tipo problema e, em $27 \%$, desconhecimento do resultado. Em relação ao auto-exame de mama, 53\% não souberam explicar o que significa e 59\% disseram não saber realizá-lo. Concluiu-se pela necessidade de veicular a informação e incrementar os métodos de comunicação a fim de facilitar às gestantes 0 acesso ao conhecimento e provocar as transformaçōes esperadas quanto ao seu comportamento em saúde.
\end{abstract}

Palavras-chave: saúde da mulher; educação em saúde; neoplasias do colo uterino; neoplasias mamárias; epidemiologia; Brasil.

*Trabalho vinculado ao Programa de Saúde da Mulher do Instituto de Estudos e Pesquisas do Cuidado à Pessoa da Universidade do Grande ABC (U niABC) e ao Programa de M estrado em Enfermagem da U niversidade de Guarulhos (UNG).

${ }^{1}$ D outora em Enfermagem, U niversidade Guarulhos (UN G). Coordenadora do N úcleo São Lucas de Assistência à Saúde da Mulher, Favela de Paraisópolis, São Paulo, SP - Brasil.

Enviar correspondência para R.A.Q.F. NZN, Alameda Jaú 186 apto 21, Jardim Paulista, 01420-000 São Paulo, SP Brasil.

2D outora em Enfermagem, Universidade do Grande ABC (U NIABC). Pesquisadora do N úcleo São Lucas de Assistência à Saúde da Mulher, Favela de Paraisópolis, São Paulo, SP - Brasil.

Recebido em setembro de 2001. 


\begin{abstract}
The gynecological cancer presents high rate of morbidity and mortality in Brazil, representing a serious public health problem, in spite of the educational campaigns and the increasing role of women in society, in seeking their rights of citizenship. The importance of this subject fosters the interest to conduct this descriptive, exploratory and quantitative study with the objective to identify how knowledgeable pregnant women are about the tests to early detect cervix uteri and breast cancer. D ata were obtained by means of an applied interview to 49 pregnant women who took part in health-promotion groups, in a poor community of the city of São Paulo. The results reveal ed that $75 \%$ of the women know the Pap test, and they associate it to detect uterine problems; that $63.3 \%$ had taken this test within a year. In $29 \%$ of the cases abnormalities were detected, and $27 \%$ had no knowledge of the results. In relation to self-breast examination, 53\% did not know what it was, and $59 \%$ said not to know how to do it. It was concluded that there is a need to disseminate information and implement communication methods to provideknowledgeto women and to stimulate them to change their behavior towards health.
\end{abstract}

Key words. women's health; health educati on; cervix neoplasms; breast neoplasms, epidemiology; Brazil.

\section{INTRODUÇÃO}

D entre os diversos tipos de neoplasia que podem afetar a população feminina, tanto 0 câncer cérvico-uterino quanto o câncer de mama destacam-se pela alta incidência e estão entreaqueles com maior índice de mortalidade nas mulheres com idade superior a 15 anos, a despeito das campanhas de prevenção e/ou detecção precoce que têm sido realizadas, especialmente em nosso país. ${ }^{1,2}$

A O rganização M undial de Saúde (O M S) indica que o câncer cérvico-uterino constituise na quarta causa de morte entre as mulheres dos países do terceiro mundo, entre eles 0 Brasil, mesmo apresentando um dos mais altos potenciais de prevenção e cura. ${ }^{3,4}$

$\mathrm{N}$ o caso do câncer de mama, ele é responsável pela morbi-mortalidade das mulheres tanto nos países desenvolvidos quanto naqueles em desenvolvimento. $\mathrm{N}$ o caso do Brasil, o câncer de mama representa a primeira causa de morte por neoplasias entre as mulheres, chegando a atingir o percentual de $16,6 \% .^{5}$

Esses dados revelam, pois, que as campanhas de prevenção e/ou detecção precoce não têm sido bem sucedidas, e esses dois tipos de câncer continuam a se constituir em sérias ameaças para a população feminina brasileira, o que pode ser explicado por diversos motivos como, por exemplo, pela dificuldade em acessar os serviços de saúde, pela demanda reprimida, pela falta de oportunidade que a mulher tem para falar sobre si e sua sexualidade e pelo desconhecimento sobre 0 câncer ginecológico. ${ }^{6,7}$

É fato que a detecção precoce do câncer cérvico-uterino e de mama é efetivada respectivamente pelo exame colpocitológico e pelo auto-exame, procedimentos de baixo custo e alta eficácia.

No caso do câncer de mama, o autoexame desde que realizado de maneira rotineira, permite identificar diversas al terações prematuramente. ${ }^{8,9} \mathrm{~N}$ esse sentido, ressalta-se a eficiência do procedimento e sua importância no decréscimo da morbimortalidade e aumento nas taxas de sobrevida e cura das mulheres. ${ }^{10}$

É importante reconhecer que 0 autoexame é considerado uma das ações preventivas do câncer de mama mais eficientes, constituindo-se, principalmente, em instrumento de transformação na medida em que propicia conscientização para os sinais iniciais da moléstia e conseqüente mudança de comportamentos e hábitos de saúde. ${ }^{7,11}$

Quanto ao exame colpocitológico ou Papanicolau, ele é reconhecidamente eficiente mas ainda não incorporado a todos os serviços de saúde, sendo sua utilização sistemática reduzida e não disponível a toda população feminina. 2,12

Estudos realizados pelo $M$ inistério da Saúde, ${ }^{13}$ estimam que apenas $7,7 \%$ das mulheres brasileiras recebem a cobertura em programas governamentais de prevenção e 
controle do câncer cérvico-uterino pelo exame colpocitológico.

$\mathrm{N}$ esse contexto sombrio, um aspecto importante a ser analisado na prevenção do câncer cérvico-uterino e de mama relacionase ao fato de que a mulher, além de esclarecimento, necessita transformar seus hábitos de saúde e envolver-se ativamente no processo, reivindicando para si o direito ao acesso e à cobertura dos programas governamentais.

T êm-se percebido que as equipes de saúde encontram dificuldade, por motivos diversos, em proporcionar às mulheres a possibilidade de realizar e/ou aprender os exames de detecção precoce. D esenvolver práticas que visem a condição de educação enquanto processo, evidencia-se como o maior problema dos profissionais que trabalham com comunidades.

N essa perspectiva, muitos dos que prestam serviços na área da saúde não têm se mostrado capazes de utilizar sua autonomia profissional para romper os laços que os ligam às ações preestabelecidas e rotineiras que, normalmente, os conduzem à insipiência na atenção aos reais problemas de uma determinada população.

Os profissionais da saúde muitas vezes justificam suas dificuldades de eficiente atuação nos processos de educação em saúde por motivos como sobrecarga de trabalho e precariedade de área física, isentando-se do comprometimento político em sua prática sobre as condições de vida da população. ${ }^{14}$ Assim, afastam-se das questões sociais e voltam sua atuação preferentemente para 0 modelos prescritivos, esquecendo-se da importância de sua participação em projetos que viabilizem a melhoria da qualidade de saúde da comunidade. ${ }^{15} \mathrm{~N}$ esse contexto, mantém-sea situação na qual não se envolvem ou não buscam conquistar espaços para realizar atendimento à mulher nos serviços de saúde a que pertencem, o que os faz deixar de assumir sua parcela de responsabilidade na melhoria das condições de saúde da população.

Considera-se que uma das formas de colaborar nessa transformação é a de realizar investigações que demonstrem as lacunas de conhecimento das mulheres sobre seu auto- cuidado e apontem caminhos que possibilitem a reversão desse quadro e, conseqüentemente, possam subsidiar programas de promoção à saúde.

Acreditando que o processo educativo deve contribuir para diminuir as estatísticas de morbi-mortalidade das mulheres, decidiuse elaborar uma série de estudos relativos à saúde da mulher, iniciando-se por esta pesquisa que tem caráter preliminar e objetiva identificar os déficits de conhecimento de mulheres sobre os exames de detecção precoce do câncer cérvicouterino e de mama, de modo a sustentar ações que influenciem a melhoria da qualidade de vida de gestantes que participam de grupos de promoção da saúde, realizado pelas autoras em uma comunidade carente.

\section{CASUÍSTICA E MÉTODO}

Trata-se de pesquisa quantitativa, descritiva e exploratória desenvolvida com gestantes participantes do N úcleo São Lucas de Assistência à Saúde da Mulher, inserido em uma comunidade carente da cidade de São Paulo.

A população foi constituída por 49 gestantes que compareceram espontaneamente e aderiram aos grupos de orientação promovidos na referida comunidade. Acrescenta-se que para participar dos grupos, todas já deveriam estar não somente matriculadas como também freqüentando o serviço de pré-natal das unidades básicas de saúde da região.

Os dados foram coletados pelas pesquisadoras durante o primeiro semestre do ano de 2000, aplicando-se questionário individualmente a todas as gestantes na ocasião em que era feita sua inscrição para composição dos grupos de orientação. 0 instrumento de coleta de dados constituiu-se deum formulário estruturado com questões abertas e fechadas referentes aos conhecimentos e hábitos de saúde relacionados aos exames de prevenção e detecção precoce do câncer cérvico-uterino e de mama.

Salienta-se que todas as gestantes concordaram em participar do estudo após a explanação de seus objetivos, obtendo-se sua autorização escrita nos termos de 
consentimento livre e esclarecido anexados aos instrumentos de coleta de dados.

Além disso, obteve-se autorização formal dos respectivos Comitês de Ética das duas instituições de ensino superior a que o N úcleo está vinculado.

\section{RESULTADOS E DISCUSSÃO}

Cerca de $80 \%$ da população estudada caracteriza-se por estruturar-se em núcleos familiares sem renda ou emprego fixos, o que demonstra o baixo nível sócio-econômico das gestantes que, além disso, possuem pouca escolaridade, uma vez que $72 \%$ estudaram apenas entre a 1 a e $5 \underline{a}$ séries e $2 \%$ são analfabetas.

Em relação ao conhecimento sobre 0 exame colpocitológico, $75 \%$ das mulheres afirmam saber o objetivo do exame. A figura 1 demonstra o nível do conhecimento autoatribuído pelas gestantes a respeito desse exame e a correlação que fazem quando questionadas sobre sua finalidade.

Figura 1. Conhecimento auto-atribuído das gestantes sobre o exame colpocitológico. São Paulo, 2000.

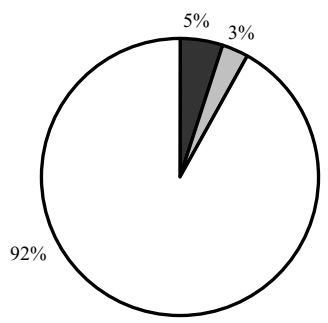

पnão conhecem a finalidade do exame

$\square$ conhecem o exame mas ignoram a finalidade

$\square$ conhecem o exame e o associam à detecção do câncer

A despeito do alto nível de conhecimento encontrado, ou seja, 92\% das respondentes afirmarem conhecer e correlacionarem corretamente a finalidade ao exame, os resultados apresentados nas figuras 2 e 3 , que demonstram a freqüência com que as gestantes realizam 0 procedimento e 0 conhecimento que têm sobre seus resultados, evidenciam a falta de orientação em relação ao exame colpocitológico.

Figura 2. Freqüência com que as gestantes realizam

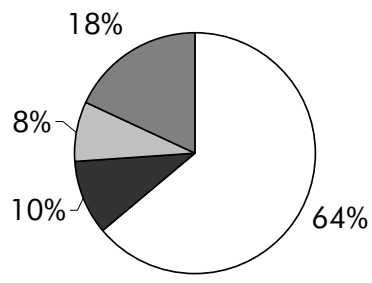

$$
\begin{aligned}
& \square \text { há um ano ou menos } \square \text { de dois a três anos } \\
& \square \text { nunca realizou } \quad \square \text { não sabe }
\end{aligned}
$$

o exame colpocitológico. São Paulo, 2000.

Figura 3. Conhecimento das gestantes sobre o resultado do exame colpocitológico. São Paulo, 2000.

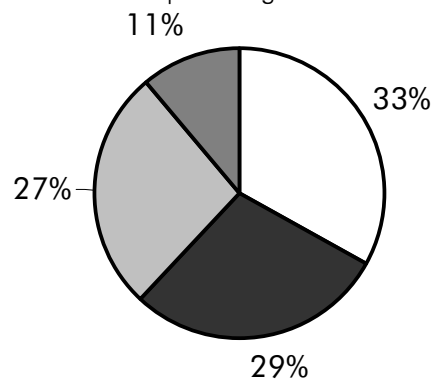

$$
\begin{aligned}
& \square \text { resultado normal } \\
& \square \text { com detecção de algum problema } \\
& \square \text { desconhece o resultado } \\
& \square \text { não recebeu o resultado }
\end{aligned}
$$

Embora 0 resultado relativo ao conhecimento das mulheres sobre o exame colpocitológico tenha sido melhor do que 0 esperado, nota-se que há necessidade de esclarecimento dessa população quanto à freqüência e importância do procedimento, uma vez que $26 \%$ nunca o realizou ou não tem informações sobre ele, mesmo realizando o pré-natal.

Q uanto ao conhecimento sobre os resultados do exame, o fato de $38 \%$ das gestantes 0 ignorarem não pode ser desconsiderado, especialmente sabendo-se que elas participam de pré-natal, oportunidade ideal para uma série de exames e informes a respeito de cuidados com a saúde.

De forma semelhante, outro trabalho realizado em comunidade carente ${ }^{11}$ indica a falta de adequada educação em saúde como um dos fatores que acarretam fal has no 
diagnóstico precoce do câncer cérvicouterino, ressaltando ser esse um fator que, associado à não implantação do programa de controle em todas as unidades básicas de saúde e à não utilização destes serviços por parte da população, acarreta graves falhas e conseqüente el evação na taxa de morbimortalidade da mulher por essetipo de agravo.

É sabido que os países que aplicam de forma contínua o método de exame colpocitológico na população obtiveram um decréscimo importante da mortalidade por câncer cérvico-uterino, fato não observado no Brasil que, pelo contrário, tem verificado aumento crescente de lesões iniciais (N IC, N IV, N IVA ou H PV) em mulheres jovens que iniciam a atividade sexual na adolescência e trocam constantemente de parceiros. ${ }^{3} \mathrm{~N}$ essa linha também deveriam se direcionar os projetos educativos em saúde, pois há necessidade de divulgar não somente a importância e finalidade do exame colpocitológico como também o uso de preservativos pelos casais e o controle dos parceiros sexuais.

Discutese aqui como fazêlo em vista da condição de subalternidade histórica das mulheres em relação aos seus companheiros sexuais, que dificilmente se engajam em projetos educativos. Esse também é o pensar de outros autores, ${ }^{6,16}$ que incluem nesse contexto discriminatório os profissionais da saúde, na medida em que centram sua atenção nos aspectos biológicos da saúde da mulher, especialmente os reprodutivos, desconsiderando a dimensão que valoriza as questões de gênero.

$\mathrm{H}$ á necessidade desses profissionais repensarem seus comportamentos de manipulação e dominação do outro, tantas vezes verificado na prática, e caminharem para um "cuidar do outro", o que implica na coexistência e na participação, na compreensão da situação única de cada mulher que procura os serviços de saúde..$^{13}$

Em relação ao auto-exame das mamas, $53 \%$ das gestantes disseram não conhecêlo quando indagadas a esse respeito, percentual que se confirmou quando eram estimuladas a dizer 0 que entendiam por esse procedimento. D as demais respondentes, $47 \%$ disseram conhecer o exame e 0 correlacionaram corretamente à sua finalidade.

A respeito da técnica de realização do autoexame das mamas, somente $41 \%$ das gestantes referiram saber executá-la referindo, em $86 \%$ das vezes, têla aprendida com profissionais da saúde. A penas 5,5\% dessas gestantes descreveram corretamente todos os passos do procedimento e as demais relataram alguns passos do método sem conseguirem explicar o porquê das etapas do auto-exame. Tal fato demonstra claramente que esse método de detecção de problemas, apesar de simples, requer, além de mudança de hábito, maior treinamento e elucidação sobre sua finalidade.

Estranha-se o fato dessas gestantes, em sua maioria, conhecerem e realizarem o exame colpocitológico mas desconhecerem o autoexame das mamas, uma vez que submeteramse a consultas ginecológicas e a exames clínicos das mamas, esperando-se que pudessem ter sido orientadas de al guma forma a esse respeito. Como sabê-lo torna-se impossível, mas alguns trabalhos ${ }^{9}$ reiteram que são muitos os ginecologistas que não examinam as mamas de suas pacientes de forma rotineira.

Aí se mostra outro problema dos serviços públicos de saúde que pouco dão atenção ao exame físico das mamas e, certamente, não ensinam autocuidado a suas clientes/ pacientes, entre eles 0 auto-exame das mamas, apesar dos inúmeros estudos que advogam pela eficácia desse procedimento rotineiro no despertar para 0 autoconhecimento fundamental nesse processo. ${ }^{17}$

Por outro lado, considera-se fundamental ressaltar que a resolução do diagnóstico precoce de câncer de mama não pode recair sobre a freqüência com que a mulher procura os serviços de saúde, linha adotada por muitas campanhas que não encontram voz na população por impor-Ihe responsabilidade sobre algo que desconhece.

Lembra-se ainda queensinar o auto-exame das mamas não é responsabilidade apenas dos profissionais inseridos nos serviços básicos de saúde, mas sim de todos aqueles que lidam com a saúde da mulher em qualquer situação. $N$ esse sentido, pesquisas ${ }^{10}$ apontam que a responsabilidade da prevenção do câncer de 
mama é de todos, independentemente da situação em que atendam a mulher.

Vale recordar, então, que profissionais da saúde do sexo feminino somente poderão participar seriamente de programas educativos na medida em que incorporarem a realização do auto-exame das mamas à sua vida pessoal, pois devem se recordar que também correm o risco de desenvolver 0 câncer de mama. A esse respeito, ressalta-se que aquelas que realizam 0 auto-exame e reconhecem sua importância têm o preparo mais que suficiente para ensiná-lo às pacientes e/ou clientes em qualquer situação, diferentemente das que não o praticam ou nele não acreditam como método eficaz de detecção de problemas. ${ }^{10,18}$

0 utro ponto que merece destaque é 0 questionamento sobre qual seria o melhor método para a difusão da prática do autoexame, não somente no Brasil como também em todo mundo, pois mesmo em países desenvolvidos parcela considerável de mulheres não se examinam, provavelmente pelo temor que o câncer nelas provoca. ${ }^{8} \mathrm{~N}$ esse contexto, deve-se pensar também em como fazer com que as campanhas de prevenção do câncer de mama possam alcançar os grupos menos privilegiados e mais suscetíveis de forma mais efetiva.

\section{CONCLUSÕES}

Todos os dados dessa pesquisa, além de caracterizarem a falta de orientação das participantes, espelham a oportunidade perdida pela equipe profissional na promoção da saúde da clientela e conseqüente melhoria da qualidade de vida da comunidade à qual presta assistência.

O s resultados evidenciam ainda que muitas mulheres acreditam conhecer os exames de detecção do câncer. Entretanto, pelo que se constatou, esse conhecimento não é suficiente ou consistente 0 bastante para influenciar positivamente nas mudanças desejáveis nas práticas de saúde. Os profissionais da área devem estar alertas para essa realidade e implementar medidas de controle da eficiência e do real impacto gerado por suas ações educativas nas populações alvo de sua atenção, pois senão estarão correndo o risco de apenas executar programas preestabelecidos sem atingir os objetivos de melhoria da qualidade da saúde.

Assim, educação em saúde não pode mais ser entendida como "algo" a ser transmitido ao cliente, menosprezando-se seu conhecimento ou acreditando-se pouco em sua capacidade de aprender ou modificar-se.

Aí se apresenta o grande desafio daqueles que, como as autoras desse trabalho, dedicam-se a tentar promover saúde em comunidades menos favorecidas, que tanto requerem em termos de ações efetivas, mas que se mostram fortemente refratárias às transformações de suas práticas de vida e de saúde, especialmente quando tratadas de forma impessoal ou apenas assistencialista.

0 atendimento voltado à clientela mais carente normalmente visa a suprir carências individuais, sem que ocorra identidade e aproximação, ou seja, o não compartilhamento da mesma causa. ${ }^{14}$ Parece claro que ao sentirse e colocar-se como superior, detentor do conhecimento, o profissional da saúde tem dificuldade em estabelecer diálogo e escutar atentamente aquilo que para ele pode parecer supérfluo ou desprovido de ciência.

A solução mais provável a este problema encontra-se na coragem em enfrentar essa situação e no desafio de trabalhar ao lado do saber popular, ${ }^{14,15}$ pois para obter retorno no processo de educação em saúde é preciso que o profissional aprenda a relativizar o poder e a sabedoria, ou seja, que perceba sua incapacidade em assegurar saúde perfeita e a necessidade de trocar verdades com a sua clientela. ${ }^{19}$

$N$ esses contextos, acrescenta-se que a abrangência do cuidar liga-se ao modo de vida próprio das pessoas e que todos devem compreender 0 fato de que cada indivíduo seleciona, concorda, decide ou opta pelos cuidados de saúde que considerar mais coerentes com o seu viver cotidiano, ${ }^{20}$ isto é, para enfrentar efetivamente os problemas, 0 profissional há que buscar junto com a população que atende soluções mais abrangentes e integradoras.

D esse modo, o conhecimento da realidade é algo premente na proposição de linhas de ação à preservação e manutenção 
de saúde. $\mathrm{N}$ ão se pode ignorar, portanto, a necessidade de veicular a informação e incrementar os métodos de comunicação com as mulheres apresentadas neste estudo de maneira a facilitar-lhes 0 acesso ao conhecimento e provocar as transformações esperadas quanto a seu comportamento em relação aos exames de detecção precoce do câncer cérvico-uterino e de mama.

O utro aspecto a ser analisado é a introdução de outras formas de orientação e de novas estratégias pedagógicas a fim de possibilitar às gestantes melhor captação e fixação dos conhecimentos, de forma a tornálas co-partícipes das ações de saúde. 0 desafio, agora, está em buscar entender o significado, os valores e as crenças que norteiam a vida dessas mulheres e que servem de esteio para suas ações de saúde.

\section{REFERÊNCIAS BIBLIOGRÁFICAS}

1. M inistério da Saúde. Secretaria $N$ acional de Assistência à Saúde. Instituto $\mathrm{N}$ acional de Câncer. Coordenação de ProgramasdeC ontrole do Câncer (Brasil). Estimativa da incidênciae mortalidade por câncer no Brasil - 2000. Rio deJaneiro: IN CA; 2000.

2. Ministério da Saúde. Instituto $\mathrm{N}$ acional de Câncer. C oordenadoria de Programa de Controle do Câncer (Brasil). 0 controle do câncer cévico-uterino edemama. Rio deJ aneiro: IN CA; 1994.

3. Alperovitch SK. Prevenção do câncer do colo do útero no ano 2000. Prat H osp 1999;|(6):401.

4. M inistério da Saúde. Instituto $\mathrm{N}$ acional de Câncer (Brasil). N ormas erecomendações do Instituto Nacional de Câncer/MS. Recomendaçõesbásicasparao controledo câncer do colo do útero no Brasil. Rev Bras C ancerol 2000;46(1):23-33.

5. M atias M . Epidemiologia. In: CostaM M , Silva H M S, D iasEN , FigueiraFilho AS. Câncer de mama para ginecologistas. Rio de Janeiro: Revinter; 1994. p.1-2.

6. FonsecaRM GS, ChiesaAM, O liveira M AC. A praxis da enfermeira na prevenção do câncer ginecológico num contexto de integração docente assistencial. Rev Esc Enf USP 1994;28(3):321-31.

7. Pinotti JA. Saúde da mulher. São Paulo:
Parâmetro; 1996.

8. Freitas Júnior R, SoaresVF, M elo N F, Andrade $M L$, Philocréon G R. Fatores determinantes do conhecimento e prática do auto-exame da mama. RBGO 1996;18(5):387-91.

9. Bromberg $S E$, Coan TCM, M elleiro $M M$, Amâncio M H , Gualda D M R, M argarido N F. Programa de conservação da saúdemamária: a importância do auto-examena detecção precoce dedoençasmamárias. Arq Cient 1997;2(4):98104.

10. H ood M D, Vargens O M C. Prevenção do câncer demama: somostodosresponsáveis. Rev Enf UERJ 1995;3(1):108-10.

11. FreitasSLF, ArantesSL, BarrosSM 0 . Atuação da enfermeira obstetra na comunidade Anhanguera, Campo Grande (M S), na prevenção do câncer cérvico-uterino. Rev Latinoam Enfermagem 1998;6(2):57-64.

12. Zeferino LC, Costa AM, M orelli M GLO, TambasciaJ, Panetta K, Pinotti JA. Programa de detecção do câncer do colo uterino de Campinas e região: 1968-1996. Rev Bras Cancerol 1999;45(4):25-33.

13. Lopes RLM . Repensando na prevenção do câncer cérvico-uterino. Rev Bras Cancerol 1996;42(4):219-25.

14. Kleba M E. Educação em saúdena assistência deenfermagem: um estudo decaso em unidade básica de saúde. In: Ramos FRS, Verdi M M , Kleba M E. Para pensar o cotidiano: educação em saúde e a práxis da enfermagem. Florianópolis: UFSC; 1999. p. 122-61.

15. Ramos FRS. 0 processo de trabalho em educação em saúde. In: RamosFRS, Verdi M M , Kleba M E. Para pensar o cotidiano: educação em saúde e a práxis da enfermagem. Florianópolis: UFSC; 1999. p. 16-61.

16. Lopes RLM, Diniz N M F, G esteira, SM A, $M$ atos M EC, Argôllo SLS, Santos ASM , et al. 0 exameginecológico para prevenção do câncer cévico-uterino: relações degênero expressas pela clientela. Rev Bras C ancerol 1999;45(4):3543.

17. D ias da Costa JS, Piccini RX, M oreira M R Avaliação da prática do auto-exame e exame físico demamasnacidadedePelotas-RS. RBGO 1995;17(6):621-32.

18. Bergamasco RB, Tsunechiro M AA. A enfermeira naprática eno ensino do auto-exameda mama. Rev Paul Enf 1993;12(3):113-21.

19. Penna CM M . Ser saudável no quotidiano da favela. Pelotas: Ed. Universitária/UFPEL; 1997. 
20. D enardin M L. Cuidando esendo cuidado: um modelo de saúde em comunidade rural. In: Gonzales RM B, Beck CLC, D enardin M L.
Cenários de cuidado: aplicação de teorias de enfermagem. Santa M aria: Pallotti; 1999. p. 159-263. 